\title{
A geometric oriented approach in drawing the simulation model of small free angular vibrations of a body
}

\author{
Petar Pavlov, Svetlana Lilkova-Markova, Borislav \\ Nakov, and Janitza Kehajova
}




\title{
A GEOMETRIC ORIENTED APPROACH IN DRAWING THE SIMULATION MODEL OF SMALL FREE ANGULAR VIBRATIONS OF A BODY
}

\author{
PETAR PAVLOV, SVETLANA LILKOVA-MARKOVA, BORISLAV NAKOV, \\ AND JANITZA KEHAJOVA
}

\begin{abstract}
The paper provides a geometry-oriented way of drawing the input block of the simulation model for studying the small free vertical angular vibrations of the body. The simulation model is created by one of the main tools of the software package MATLAB SIMULINK. The geometry of the input block of the simulation model looks like the geometry of the dynamic model, allowing easy and attractive introduction of various geometric, inertial, elastic and viscous characteristics. The model described in the paper is made with a licensed version of the software package MATLAB, purchased with funds from a research project on which the authors of the paper have worked over the last two years.
\end{abstract}

2000 Mathematics Subject Classification: 34B10, 34B15

Keywords: dynamic model, mathematical model, simulation model, small angular vibrations, elastic-viscous characteristics

\section{INTRODUCTION}

The input block for which geometry-oriented approach to creation is offered is part of a generalized simulation model for studying small free vertical angular vibrations of the body. The standard structure of a similar model to study the dynamic behavior of any physical object consists of two parts:

$\diamond$ A system of connected blocks to integrate the deferential equation describing the movement of the object;

$\diamond$ A system of blocks for visualizing the results.

A standard simulation model of an analysis of the dynamics of a material object or a system with one degree of freedom is shown on Figure 1, but without the left input block, which will be discussed below.

The characteristics of the material objects or the system and the relationships limiting their movement are usually given by a separately compiled text file produced by the programming language of the software package MATLAB [1]. Prior to starting the simulation model, a input file for the mentioned characteristics should be started. 


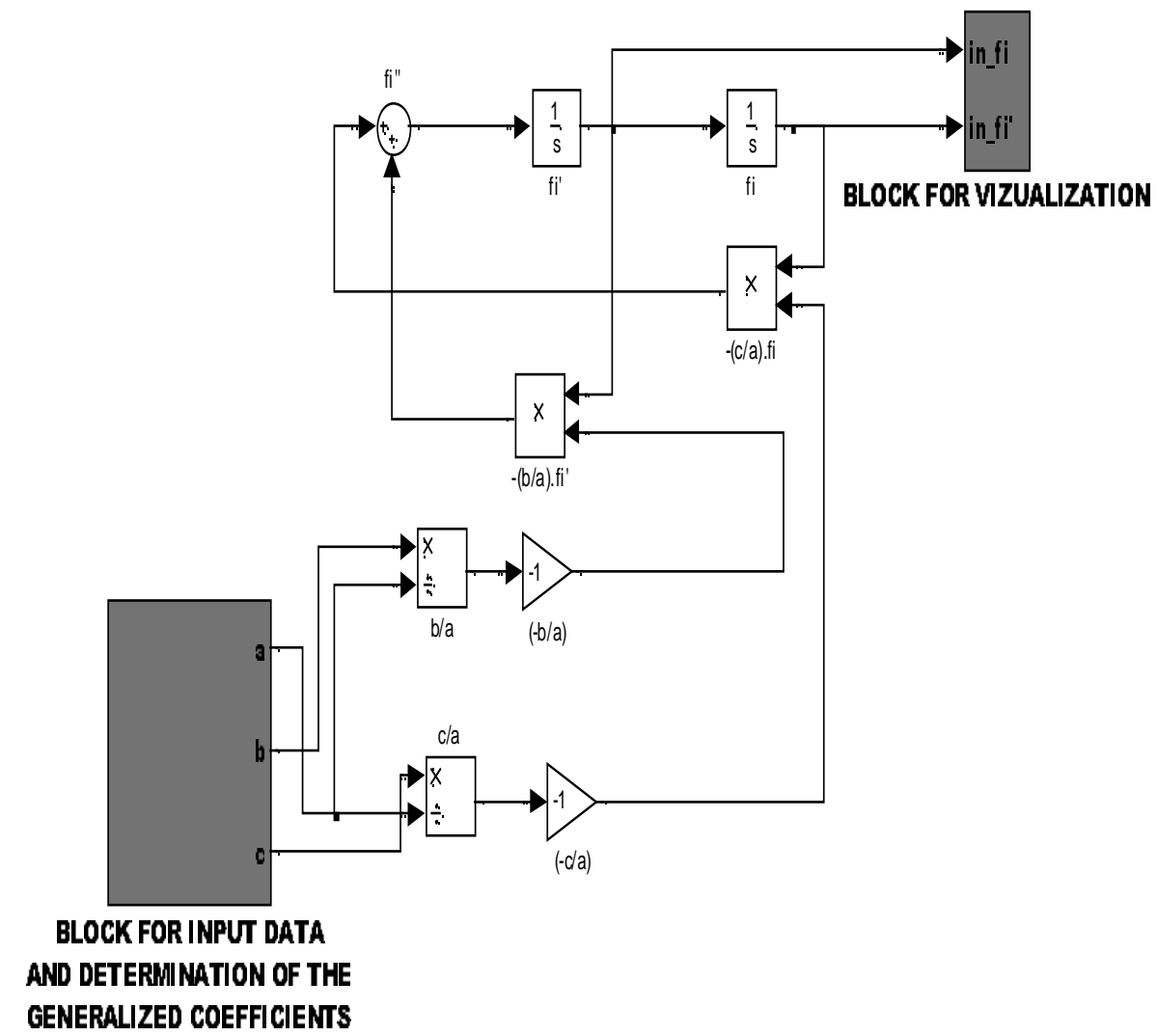

FIGURE 1. A simulation model for studying the dynamics of a vibrating system with 1 DOF.

This naturally leads to a number of inconveniences. Each exit from the system imposes a new startup of the text file or of a file, saving the previously entered input data. Many inconveniences appear when changing one or several inputs. It is not easy to check the value of the individual characteristics, especially if several files with similar names of the individual variables are open in the working window of the program. The text and the simulation file should always be transferred together. The absence of one of them would prevent the right solution of a dynamic problem.

All these inconveniences in the paper can be removed by establishing an additional block of input data. This approach is implemented when testing the small free angular oscillation of a body, but can also be applied to the forced oscillations. The architecture of the block, composed in the middle of the SIMULINK, resembles the dynamic model for the study of the small angular vibrations of the body [2]. It allows 
an easy and convenient entry or modification of the physic-mechanical characteristics of the vibrating system.

\section{Presentation}

The construction of a simulation model is the third stage in studying the dynamics of an oscillating system. Prior to this, an idealized dynamic model is created along with a corresponding mathematical model of the vibrations. The dynamic model of small angular vibrations of a body in the vertical plane is described in [2], and the deductively derived model of free ones is shown on Figure 2.

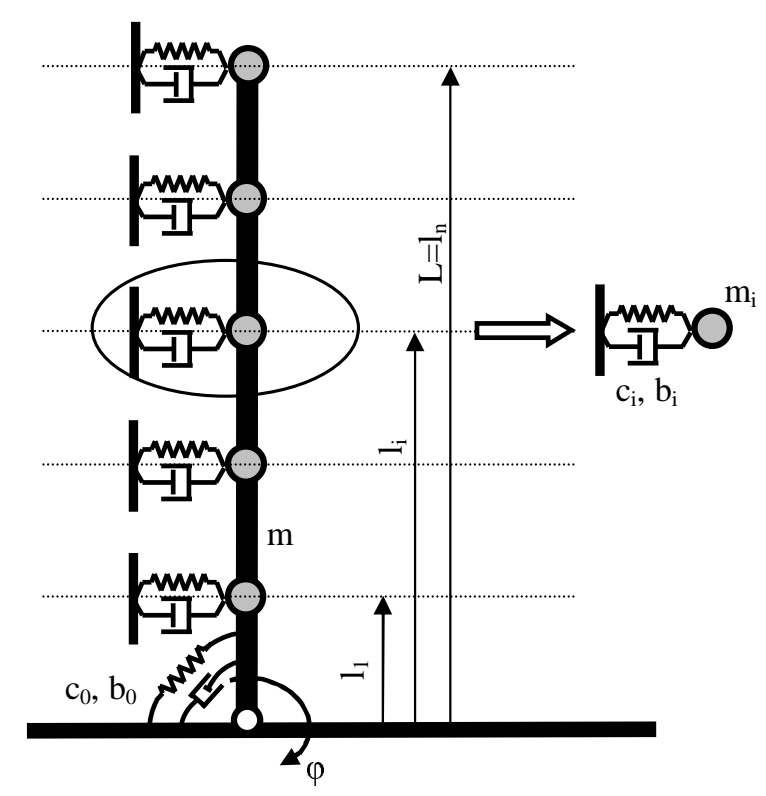

FIGURE 2. Dynamic model for free small angular vibrations of a body in the vertical plane.

The corresponding mathematical model, obtained by the Lagrange equations of second class, in a generalized coordinate (the angle), in which the vibrating body deviates from the vertical axis, is

$$
a \ddot{\varphi}+b \dot{\varphi}+c \varphi=0 .
$$

In the equation, $a, b$ and $c$ denote the generalized inertial, the dissipative, and the elastic coefficients respectively.

The general appearance of the simulation model for analyzing the small free angular vibrations of a body is that of Figure 1, plus the block of input data. Like any standard simulation model for analyzing the dynamics of systems with one degree of 
freedom, it contains the basic blocks - block for integration of the differential equation and block for visualization of the results. The unit of integration shows that the generalized coefficients $a, b$ and $c$ must be known.

In the standard simulation programs, they can be found through the input program, because they are a function of the input data by the formulas

$$
\begin{aligned}
a & =\frac{1}{3} m \ell^{2}+\sum m_{i} \ell_{i}^{2}, \\
b & =b_{0}+\sum b_{i} \ell_{i}^{2}, \\
c & =c_{0}+\sum c_{i} \ell_{i}^{2}-g\left(m \frac{\ell}{2}+\sum m_{i} \ell_{i}\right) .
\end{aligned}
$$

As mentioned in the paper, the compilation of an additional file is ignored and, instead of that, a block for input data and for calculating the coefficients by the formulas (2.2) is added. The idea is that the incoming block should be similar to the real dynamic model. This is why the blocks are in a form resembling the respective elements of the vibrating system. The main part of this input block of the simulation model of a system consisting of a vibrating body and two concentrated masses is shown on Figure 3.

The type of all blocks in the subsystem is constant because the values of the parameters they introduce are constant too. The name of each block contains the parameter, which is also related to equality sign. By clicking on the desired block, the corresponding window opens, and the respective characteristic can be entered or confirmed. The entered characteristics appear as values in the center of each block. The units in which each characteristic is entered, can be written in a specific location in the subsystem. By default, the base units in the system SI are used for length, mass, spring stiffness and a linear coefficient of resistance damper.

The blocks for the geometric characteristics are vertically elongated rectangles reminding of the measurement lines in the dynamic model. The block for mass of the vibrating rod is also vertically extended rectangle, so that it can match the rod in the dynamic model. The blocks for entering the concentrated masses are adjoined to the last block squares. The blocks for entering the characteristics of the elastic-viscous sets along the length of the body are complemented by blocks in the form of spring and damper.

Output block with a certain number is attached to each constant block type. The numbers of the outputs are used as inputs for the next subsystem - the subsystem for calculation of the generalized coefficients of equations (2.1) and (2.2).

\section{CONCLUSiON}

The compilation of a separate module for data entry in the construction of a simulation model, for the study of the dynamics of an oscillating system, has the following advantages in comparison with the standard simulation model: 


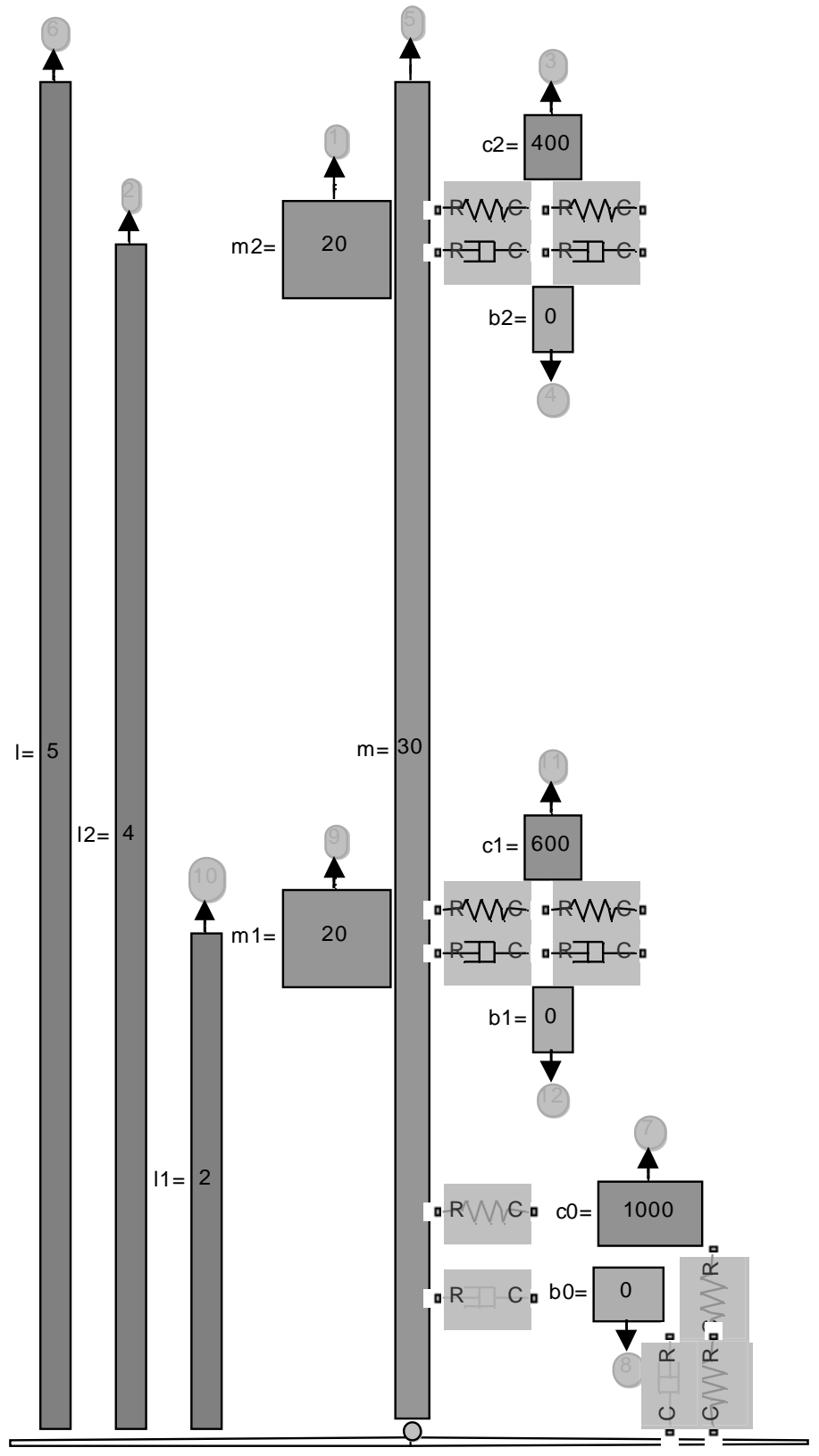

FIGURE 3. Part of the input block of the simulation model for analyzing the free small angular vibrations of a body in vertical plane. 
$\diamond$ When entering data, the user sees the real dynamic model in the individual components in which he enters or changes certain parameters;

$\diamond$ It is easy to solve the problem of optimal dynamic synthesis.

The model described in the report is made with a licensed version of the software package MATLAB, purchased with funds from a research project in which the authors of the paper have worked in the last two years.

\section{ACKNOWLEDGEMENT}

The authors of the report express their gratitude to the Centre of the Research and Design in the UACEG, Sofia, for the financial assistance for purchasing the licensed version of the software package MATLAB, thanks to which the described research was carried out.

\section{REFERENCES}

[1] A. Ivanov, Modeling if dynamic problems with MATLAB. Sofia: Arte Novo, 1985.

[2] S. Lilkova-Markova, P. Pavlov, B. Nakov, and J. Kehajova, "Generalized dynamic and mathematical model of small angular vibrations of the body, rotation in the vertical plane," in Proceedings of the International jubilee scientific conference "Science \& Practice", vol. 4. Sofia: UACEG, 2012, pp. 117-122.

Authors' addresses

\section{Petar Pavlov}

UACEG, Technical Mechanics Dept., 1 Smirnenski Blvd., 1046 Sofia, Bulgaria

E-mail address: pdp_mech_fhe@uacg.bg

Svetlana Lilkova-Markova

UACEG, Technical Mechanics Dept., 1 Smirnenski Blvd., 1046 Sofia, Bulgaria

E-mail address: lilkova_fhe@uacg.bg

\section{Borislav Nakov}

UACEG, Technical Mechanics Dept., 1 Smirnenski Blvd., 1046 Sofia, Bulgaria

E-mail address: nakov_fhe@uacg.bg

\section{Janitza Kehajova}

UACEG, 1 Smirnenski Blvd., 1046 Sofia, Bulgaria

E-mail address: dreaming_girleabv.bg 